\title{
Swabbing the Urban Environment - A Pipeline for Sampling and Detection of SARS-CoV-2 From Environmental Reservoirs
}

\author{
Maria I. Rojas ${ }^{1,2}$, Steven S. Giles ${ }^{3}$, Mark Little ${ }^{1,2}$, Rafael Baron ${ }^{1,2}$, Isabella Livingston ${ }^{1,2}$, Taylor R. T. Dagenais ${ }^{3}$, Jason \\ Baer $^{1,2}$, Ana G. Cobián-Güemes ${ }^{1,2}$, Brandie White ${ }^{1,2}$, Forest Rohwer ${ }^{1,2}$ \\ ${ }^{1}$ Department of Biology, San Diego State University ${ }^{2}$ Viral Information Institute, San Diego State University ${ }^{3}$ Big Rose Web Design, LLC
}

\section{Corresponding Author}

Maria I. Rojas

mariaisabelrm@gmail.com

\section{Citation}

Rojas, M.I., Giles, S.S., Little, M.,

Baron, R., Livingston, I.,

Dagenais, T.R.T., Baer, J.,

Cobián-Güemes, A.G., White, B.,

Rohwer, F. Swabbing the Urban Environment - A Pipeline for Sampling and Detection of SARS-CoV-2 From Environmental Reservoirs. J. Vis. Exp. (170), e62379, doi:10.3791/62379 (2021).

\section{Date Published}

April 9, 2021

DOI

$10.3791 / 62379$

URL

jove.com/video/62379

\section{Abstract}

To control community transmission of severe acute respiratory syndrome coronavirus 2 (SARS-CoV-2) during the 2020 global pandemic, most countries implemented strategies based on direct human testing, face covering, and surface disinfection. Under the assumption that the main route of transmission includes aerosols and respiratory droplets, efforts to detect SARS-CoV-2 in fomites have focused on locations suspected of high prevalence (e.g., hospital wards, cruise ships, and mass transportation systems). To investigate the presence of SARS-CoV-2 on surfaces in the urban environment that are rarely cleaned and seldomly disinfected, 350 citizens were enlisted from the greater San Diego County. In total, these citizen scientists collected 4,080 samples. An online platform was developed to monitor sampling kit delivery and pickup, as well as to collect sample data. The sampling kits were mostly built from supplies available in pandemic-stressed stores. Samples were processed using reagents that were easy to access despite the recurrent supply shortage. The methods used were highly sensitive and resistant to inhibitors that are commonly present in environmental samples. The proposed experimental design and processing methods were successful at engaging numerous citizen scientists who effectively gathered samples from diverse surface areas. The workflow and methods described here are relevant to survey the urban environment for other viruses, which are of public health concern and pose a threat for future pandemics.

\section{Introduction}

SARS-CoV-2 is thought to be mainly transmitted via the inhalation of contaminated aerosols and droplets from direct contact with infected individuals ${ }^{1,2,3,4}$. However, during the initial phases of the global COVID-19 pandemic, efforts to control transmission of SARS-CoV-2 focused strongly on disinfecting surfaces, handwashing, and sanitization. By 
the end of 2020, transmission guidelines from the World Health Organization $(\mathrm{WHO})^{5}$ and the U.S. Centers for Disease Control and Prevention $(C D C)^{6}$ deemed airborne transmission a hazard mainly when in close contact $(<2$ m) with an infected person or in the presence of aerosolgenerating medical procedures. Self-inoculation after contact with contaminated surfaces or inhalation of aerosolized fomites have yet to be ruled out as a route of transmission of SARS-CoV-2.

COVID-19 cases have been reported where airborne transmission seems unlikely 7,8 . SARS-CoV-2 virions remain infectious on copper for up to $8 \mathrm{~h}$, on cardboard and stainless steel for up to $24 \mathrm{~h}$, and on plastic for up to $48 \mathrm{~h}^{9}$. In cruise ship cabins, SARS-CoV-2 RNA was detected 17 days after the passengers had departed ${ }^{7}$. Air and surface samples from hospitals and mass-transit systems have tested positive for SARS-CoV-2 and other coronaviruses $8,10,11,12,13,14$. A study performed on the outer packaging of Halloween candy handled by asymptomatic and moderate/mildly symptomatic COVID-19 patients, concluded that the combination of hand washing by the handler and washing of the candy with hand soap reduced SARS-CoV-2 RNA to below threshold levels ${ }^{15}$.

Several methods for SARS-CoV-2 diagnostics have been published based on real-time reversetranscription polymerase chain reaction (RTqPCR $)^{16,17}$, reverse-transcription loop-mediated isothermal amplification (RT-LAMP) $)^{11,18,19,20,21}$, and CRISPR-Cas technologies $18,19,22,23$. Most require RNA extraction kits that are often in short supply during periods of significant global demand, and very few have been used for environmental screening of the virus ${ }^{24}$. The detection of SARS-CoV-2 RNA using RT-LAMP has been demonstrated to be over $83 \%$ concordant to using RT-qPCR. Furthermore,
RT-LAMP resulted in $25 \%$ reduction in inconclusive results compared to RT-qPCR ${ }^{15}$.

RT-LAMP is a simple technique that uses a reverse transcriptase to synthesize cDNA from an RNA template ${ }^{25}$, followed by a DNA polymerase with strong stranddisplacement activity that synthesizes DNA at constant temperature (i.e., isothermal amplification) ${ }^{26}$. Higher specificity of viral genome detection is achieved by using four or six primers that recognize six or eight regions of the target DNA. Amplification is initiated from an internal primer and yields a semi-double-stranded DNA structure. The leading strand is then amplified by an outer primer. These amplifications are repeated for the reverse primers. Internal and outer primers on either end have an internal reverse self-complementary site that forms a loop in the amplification product ${ }^{26,27}$. In isothermal strand displacement, asynchronous DNA synthesis generates high quantities of amplified product where continuous polymerization amplifies the signal of as few as 10 copies per reaction $11,20,28$. The colorimetric RT-LAMP mix is weakly buffered and uses phenol red as a $\mathrm{pH}$ indicator. As the polymerase incorporates a nucleotide, it releases a proton, and enough protons will change the $\mathrm{pH}$ of the solution as well as its color from pink to yellow $11,20,28,29$.

RT-LAMP was developed for the detection of mosquito-borne diseases in peripheral health care facilities that lack fully equipped laboratories ${ }^{25}$ and for the rapid detection of other RNA viruses like human immunodeficiency virus ${ }^{30}$. The most vulnerable populations in epidemic outbreaks-as per the WHO definition-often lack sufficient economic resources and the appropriate equipment to carry out detection (United Nations Global Public Health Agenda). In the current SARSCoV-2 pandemic, supplies such as medical-grade swabs and 
reagents for RNA extraction kits have not been able to meet global demand, especially in non-manufacturing countries. The proposed protocol used a guanidinium thiocyanate (GITC)-based crude RNA extraction, which effectively preserved the RNA in a cold-chain independent manner and significantly reduced the persistence of inhibitors from the sample. Moreover, the GITC-chloroform extraction protocol is based on the separation of RNA from DNA and proteins followed by the respective precipitation, allowing recovery of most of the genetic material. These advantages outweigh the potential hazards of citizen scientists handling the chemical if measures are taken to appropriately inform them of the risks.

The proposed workflow uses materials and reagents that are of general use. It requires equipment that is available in basic, often rural, laboratory settings. These methods are inexpensive, highly resistant to inhibitors often found in environmental samples or samples that cannot be processed with extraction kits, and eliminate the need for a highprecision thermocycler. This study presents a pipeline for sampling and detection of SARS-CoV-2 from environmental reservoirs on commonly touched and rarely disinfected surfaces of households and the urban environment.

\section{Protocol}

See the Table of Materials for a detailed list of reagents and supplies, including catalog numbers, manufacturer, and corresponding costs.

\section{Sampling the urban environment}

\section{Citizen scientist outreach}

1. Recruit citizen scientists using a direct and clear call-to-action released via local and social media.
Create a social media handle (e.g., \#swab4corona) to connect the topic across social media content.

2. Create an email account for direct communication between the laboratory team and each citizen scientist, managed by a person fluent in the main languages of the region of interest (e.g., Spanish and English for San Diego County).

3. Build a secure web-based sample management platform (SMP) to serve as a database, a laboratory information management system (LIMS), and to communicate with citizen scientists.

NOTE: The SMP provides a centralized location where users request a kit, access sample collection protocols, submit sample metadata, and request a pickup for completed sample kits.

4. Create a link to the SMP (e.g., https:// demo.covidsample.org/) (Figure 1) for individuals to apply to participate in the environmental sampling effort by answering biosafety-related questions specified in an online form.

5. Secure access to the SMP using an Authentication Application Programming Interface facilitated by a cloud computer service provider. Give approved users access to the SMP.

NOTE: Refer to literature for a description of the OAuth 2.0 protocol $^{31}$ for authentication and authorization. It provides a frictionless sign-in process for citizen scientist volunteers. It also allows users to sign-in with an existing account, eliminating the need to create a custom sign-in solution and manage user credentials, which saves a significant amount of time and encourages participation. According to recent reports, the free email service 
available for the chosen cloud computer service provider has approximately 1.5 billion monthly-active email users; requiring an email account from this service provider for participation is not considered a discouraging factor.

6. Explain the objective of the study and biosafety considerations to the citizen scientists at the SMP before they request their first kit. Provide a multilingual plugin to enable navigation in any of the languages available from a multilingual neural machine translation service facilitated by a cloud computer service provider.

7. Include in the sampling section graphic and audiovisual protocols in English and Spanish.

8. Assign a unique identifier to each kit, and design the user interface to utilize buttons linked to Sample ID to streamline the data entry process (Figure 1A).

9. Use a delivery route planning software with a mobiledevice application to be used by drivers to optimize delivery/pickup routes and notify citizen scientists of accurate estimated times of arrival.

10. Build the LIMS platform on a PHP web service stack, and host it on a commercial hosting platform (suggested operating system, web server software, and database software are specified in the Table of Materials).

11. Provide a secure web-based application interface to enable lab personnel to manage data quickly and easily in the LIMS. Provide data visualization using a data Charting Application Programming Interface facilitated by a cloud computer service provider.

12. Visualize geospatial data using a geospatial application programming interface facilitated by a cloud computer service provider. Store the data submitted to the LIMS through the SMP to facilitate (1) centralized storage of project data; (2) tracking of sample/data processing workflows; and (3) management of the logistics of sample kit distribution to citizen scientists.

13. Secure submitted metadata using best practices (e.g., https://demo.covidsample.org/).

14. Pre-load information such as Sample Kit ID, Sample ID, date, time, and Global Positioning System (GPS) coordinates (automatically gathered from a picture of the site) to enable data-type compliance and minimize the submission of erroneous or missing data by the user (Figure 1B). Include the following fields to be manually and swiftly ( $<1 \mathrm{~min}$ ) filled by the citizen scientist: date and time of collection, a brief description of the location, and a picture of the sampling site.

15. Sanitize all uploaded data, and validate for data type. For example, validate image data uploaded by users to select .jpg files, rename them with Sample ID for fast association with the sample, and store uploaded images in a separate secure location not accessible to users.

16. Activate the option to request kit delivery and pickup when all samples (16) have been completed. Additionally, activate the option to request a new kit to be delivered upon pickup of the previous one (Figure 1A).

NOTE: For volunteers who prefer a non-web-based platform and for those worried about disclosing their GPS location (e.g., members of the community concerned about their migratory status), kits can 
be delivered at an agreed meeting location and volunteers asked to record a written version of the data collection. For communication between the laboratory and each citizen scientist, have a bilingual member of the project available for telephone calls and texts.

\section{Swab for Corona}

1. Identify an epidemiologically relevant time window for the sampling effort.

2. Build a kit that contains all the sampling supplies, including the necessary personal protective equipment (i.e., mask, gloves), a sampling protocol, and biosafety relevant information (Figure 2). Prelabel each tube with the assigned unique identifier (Sample ID).

3. Swab rarely disinfected surfaces that are exposed to aerosolized fomites in households and the urban environment.

1. Wear the provided mask in public and a new pair of gloves for the collection of each sample to avoid cross-contamination. After finishing the sampling, use the provided hand sanitizer.

2. Wet a $1 \mathrm{~cm}^{2}$ polyester absorbent swab (e.g., mop pads) with a detergent (e.g., $0.5 \%$ sodium dodecyl sulfate (SDS)) to inactivate the virus by disrupting its envelope and to stabilize the naked RNA by inducing unfolding of RNases ${ }^{32}$.

3. Swab a surface of $10 \mathrm{~cm}^{2}$. Aided by a toothpick, completely submerge each sample swab in the corresponding pre-labeled tube containing 200 $\mu \mathrm{L}$ of guanidinium thiocyanate solution (GITC). Store tubes at $4{ }^{\circ} \mathrm{C}$ until they are transported to the laboratory. Once the samples arrive to the laboratory, store them at $-80{ }^{\circ} \mathrm{C}$.

NOTE: GITC is a toxic irritant; avoid contact with skin. GITC solution is simple to prepare from common laboratory chemicals, for the recipe $\mathrm{see}^{33,34}$. It inactivates the virus, stabilizes RNA by denaturing RNases ${ }^{34,35,36}$, and stabilizes samples at room temperature. The kit, however, includes ice packs to keep the samples cold without the need to use household refrigerators for storage.

\section{SARS-CoV-2 detection}

1. Total RNA isolation

1. Disinfect surfaces, equipment, and pipettors with a solution of $2 \mathrm{mM}$ copper sulfate and $3 \%$ hydrogen peroxide; followed by a solution of $10 \%$ bleach, 90 $\mathrm{mM}$ sodium bicarbonate, $5 \%$ SDS, and $2.5 \% \mathrm{NaOH}$. Wipe thoroughly with distilled water followed by $75 \%$ ethanol.

NOTE: These solutions are an alternative to the commercially available solutions.

2. Thaw samples on ice. Vortex samples for 2 min at medium speed.

3. To increase the speed of the screening, process the samples in pools. If a pool is positive, extract the RNA of each sample independently to find the positive sample/s. Combine the samples from each sampling kit (16 total) into 2 pools of 8 samples.

NOTE: Having 8 samples per pool means that only 2 pools need to be processed per kit. If a pool is positive, then the individual samples are re- 
processed for individual RT-LAMP analysis. This reduces time, costs, and reagents.

4. Pool $50 \mu \mathrm{L}$ of each of 8 samples into a microcentrifuge tube (total volume $400 \mu \mathrm{L}$ ); save the remaining sample at $-80{ }^{\circ} \mathrm{C}$. Add 0.2 volumes (80 $\mu \mathrm{L}$ ) of chloroform, vortex for $15 \mathrm{~s}$, and then incubate for $20 \mathrm{~min}$ at $4{ }^{\circ} \mathrm{C}$. Centrifuge at $13,000 \times g$ for 20 $\min$ at $4{ }^{\circ} \mathrm{C}$.

5. Transfer the aqueous (clear liquid) layer into a new microcentrifuge tube. Store the remaining interface and pink liquid in the $-80^{\circ} \mathrm{C}$ freezer; these fractions contain DNA and proteins ${ }^{33,36}$.

6. Add an equal volume of isopropanol $(\sim 200 \mu \mathrm{L})$ and $2.6 \mu \mathrm{L}$ of glycogen coprecipitant $\left(15 \mathrm{mg} \mathrm{mL}^{-1}\right)^{37}$. Mix well, and incubate at $-20^{\circ} \mathrm{C}$ for at least $1 \mathrm{~h}$, followed by $4{ }^{\circ} \mathrm{C}$ for $10 \mathrm{~min}$ to precipitate RNA.

NOTE: The protocol can be paused here by incubating samples at $-20^{\circ} \mathrm{C}$ overnight instead of 1 h.

7. Centrifuge at $13,000 \times g$ for $20 \mathrm{~min}$ at 4 ${ }^{\circ} \mathrm{C}$. Remove the supernatant without disturbing the pellet. Resuspend the pellet in $50 \mu \mathrm{L}$ of diethylpyrocarbonate (DEPC)-treated water, and add an equal volume $(50 \mu \mathrm{L})$ of RNase-free $5 \mathrm{M}$ ammonium acetate and 2.5 volumes $(250 \mu \mathrm{l})$ of $100 \%$ ethanol $^{7,38}$.

NOTE: Ammonium ions inhibit polynucleotide kinase if used in a downstream process ${ }^{38}$. The mix precipitates RNA while leaving deoxynucleoside triphosphates and oligosaccharides in solution ${ }^{38}$.

8. Mix well, and incubate at $-20^{\circ} \mathrm{C}$ for at least $1 \mathrm{~h}$, followed by $4{ }^{\circ} \mathrm{C}$ for $10 \mathrm{~min}$ to precipitate RNA.
NOTE: The protocol can be paused here by incubating samples at $-20^{\circ} \mathrm{C}$ overnight instead of 1 h.

9. Centrifuge at $13,000 \times g$ for $20 \min$ at $4{ }^{\circ} \mathrm{C}$. Wash the pellet with $1 \mathrm{~mL}$ of cold $\left(-20^{\circ} \mathrm{C}\right)$, freshly made $75 \%$ ethanol. Centrifuge at $8,000 \times g$ for $5 \min$ at $4{ }^{\circ} \mathrm{C}$. Remove the supernatant with a P10 pipette to avoid disturbing the pellet.

10. Air dry the pellet for $10-15$ min until there is no remaining ethanol. Resuspend the pellet in $50 \mu \mathrm{L}$ of DEPC-treated water, add $5 \mu \mathrm{L}$ of $10 x$ DNase buffer $+1 \mu \mathrm{L}$ of DNase $\left(2\right.$ Units $\left.\mu \mathrm{L}^{-1}\right)$, and incubate at 37 ${ }^{\circ} \mathrm{C}$ for $30 \mathrm{~min}$.

11. Add 0.1 volumes $(5.6 \mu \mathrm{L})$ of DNase inactivation reagent, incubate at room temperature for $5 \mathrm{~min}$, and mix gently every minute. Centrifuge at $13,000 \times g$ for 2 min, and transfer supernatant to a new tube $(\sim 50 \mu \mathrm{L})$. Place the tube on ice immediately while preparing the RT-qPCR or RT-LAMP reactions, or store in a $-20{ }^{\circ} \mathrm{C}$ freezer.

NOTE: Perform RNA Isolation in an amplicon-free room to avoid carry-over contamination.

2. Multiplex reverse-transcription loop-mediated isothermal amplification (RT-LAMP)

1. Prepare a 20X primer mix solution (Table 1) for each set of primers (Table 2). Prepare the RT-LAMP reaction mix (Table 3 ) at room temperature with $10 \%$ excess volume to account for pipetting loss.

NOTE: The colorimetric LAMP $2 \mathrm{X}$ master mix with Antarctic thermolabile uracil-DNA glycosylase (UDG) prevents amplification of DNA contamination from previous reactions ${ }^{20,28}$. 
2. Vortex and spin down the mixture. Dispense $20 \mu \mathrm{L}$ of the mix into each reaction tube: sample, spiked, positive control, and negative control. Incubate the reactions in the tubes at room temperature for 10 min to allow the UDG to act on potential carry-over contamination.

3. Add $5 \mu \mathrm{L}$ of RNA to the sample reaction, $5 \mu \mathrm{L}$ of RNA + $2.5 \mu \mathrm{L}$ (450 copies) of synthetic SARS-CoV-2 RNA to the spiked reaction, $2.5 \mu \mathrm{L}$ (450 copies) of synthetic SARS-CoV-2 RNA to the positive control reaction, and $5 \mu \mathrm{L}$ of $\mathrm{H}_{2} \mathrm{O}$ to the negative control reaction. Mix well, and spin down the reactions; thaw all RNA on ice.

4. While the thermocycler, or the water bath, is heated to $65{ }^{\circ} \mathrm{C}$, allow all reactions to remain at room temperature. The UDG will be inactivated at $>50$ ${ }^{\circ} \mathrm{C}$. Place the reactions in the thermocycler (use heat lid), incubate at $65{ }^{\circ} \mathrm{C}$ for $40 \mathrm{~min}$, and let the reactions reach room temperature $\left(\sim 22{ }^{\circ} \mathrm{C}\right.$ for 5 $\min$ ), or cool on ice for $1 \mathrm{~min}$. Analyze the results using the colorimetric feature (simple observation) or by running the products in an agarose gel.

NOTE: Although the limit-of-detection (LOD) is 10 copies per reaction, the frequency of detection increases as the copy number approaches 500 copies per reaction (Figure $\mathbf{3 A}$ ). For colorimetric observation, note that a negative result is indicated by pink $(\mathrm{pH}=8.8)$, whereas a positive result is indicated by yellow $(\mathrm{pH}=5)$ (Figure 3B). The colorimetric option avoids the opening of the RTLAMP tubes after amplification, which will reduce the volume of the RT-LAMP products in the working environment and carry-over contamination. For gel electrophoresis, prepare a $1.5 \%$ agarose gel with
1X DNA gel stain in $0.5 \%$ Tris/Borate/EDTA (TBE) buffer. Load $25 \mu \mathrm{L}$ of the reaction $+5 \mu \mathrm{L}$ of $6 \mathrm{X}$ loading dye in each well. Run the gel at $100 \mathrm{~V}$ for 60 min. A molecular marker is not needed as positive samples show a ladder pattern (Figure 3C).

\section{Representative Results}

Samples collected by citizen scientists for the detection of SARS-CoV-2. During an 8-month period (mid-March to the third week of November 2020), 482 citizens were approved to participate in this project, of which 350 (73\%) requested a kit. A total of 362 kits were delivered (i.e., some participants requested multiple kits), and 246 (70\%) were returned (Figure 4A,B). All 4,080 samples contained in these kits were processed. Collection sites were distributed across North Coastal, North Central, Central, and Southern districts of the county, as well as a few at the eastern districts (Figure 4A). These districts have the highest population density of San Diego County and the most documented COVID-19 cases, as reported by the San Diego Human Health Service Agency ${ }^{39}$.

Citizens requested pickup of most sampled kits (i.e., average success rate: $70.4 \%$ ). Each day, 1-16 kits were requested, and 0-14 kits were returned to the laboratory (Figure 4B). A survey of the citizen scientists showed that the collection of a complete kit (16 samples) took 1-3 h distributed throughout an average of 8 days (Figure 4A and Table 4). The great majority of the kits were complete $(91.1 \%)$, meaning they contained a swab inside all 16 sample tubes, and the corresponding sampling data was uploaded to the LIMS (Figure 4A and Table 4).

Detection of SARS-CoV-2 using GITC-chloroform extraction and multiplex reverse transcriptase loop- 
mediated isothermal amplification (RT-LAMP). For the colorimetric RT-LAMP assay, two sets of primers $11,20,28$ were used to target the nucleocapsid (N2) and the envelope (E1) genes (Table 2). The sequences recognized by these primers are in the same region as the primers and probes approved by the $\mathrm{CDC}^{40}$ and the European Union (EU) $)^{41}$ for human diagnosis of COVID-19 by RT-qPCR. These results corroborate what Zhang et al. ${ }^{28}$ described, in which adding $60 \mathrm{mM}$ guanidine hydrochloride to the reaction increases the LOD when run in multiplex. The LOD at a frequency of 100\% was 500 copies per $25 \mu \mathrm{L}$ reaction (Figure 3A,B). In the colorimetric RT-LAMP, positive samples changed color from pink to yellow due to a $\mathrm{pH}$ shift from $\sim 8$ to 5.5 (Figure 3B). When the reaction turned orange at low-copy numbers, samples were run in a $1.5 \%$ agarose gel to confirm these were positive and resulted in a ladder-like pattern (Figure 3C). RT-LAMP was used to detect SARS-CoV-2 in pooled RNA samples.

To control for false negatives due to reaction inhibitors, each sample was tested in an additional reaction spiked with 500 copies of synthetic SARS-CoV-2. Positive pooled samples were dereplicated by isolating the RNA of each individual sample in the pool and run in a RT-LAMP reaction to determine the identity of the positive sample. The detection results were then uploaded to the LIMS where the unique sample ID was paired with the information on date, time, GPS coordinates, site, and image of the sample.

\section{Real-time and traditional RT-PCR methods: inhibition by} sample contaminants. To select the best method suitable for the proposed detection pipeline, other RNA amplification methods were tested with environmental samples collected by a pilot cohort of citizen scientists. Examples of the results of each of these methods are presented in Figure 5 to depict their sensitivity to environmental inhibitors and background signal noise at low viral copy-number concentrations.

Six RT-qPCR formulations (Table of Materials) approved by the CDC and the WHO were tested for detection of the virus on environmental samples. Protocols were followed according to the manufacturer's instructions as well as CDC guidelines for the detection of SARSCoV-2 in clinical settings ${ }^{40}$. Reactions containing different concentrations of synthetic SARS-CoV-2 RNA controls were spiked into swabbed-surface samples after crude RNA isolation. All master mixes were sensitive to inhibitors at LOD concentrations of the positive control (Figure 5A).

To bypass inhibitors of these real-time technologies, a traditional RT-PCR system was tested. A one-step RTPCR system (Table of Materials) was used to amplify the nucleocapsid gene using the primer sets $N 1, N 2$, and the envelope gene using the primer set $E 1$ approved by the CDC (USA) and the ECDC (EU), respectively (Table 2).

Protocols were followed according to the manufacturer's instructions as well as CDC guidelines for the detection of SARS-CoV-2 in clinical settings ${ }^{40}$. The primer sets $N 1$ and $N 2$ designed by the CDC yield a $\sim 70$ bp product; however, low-copy number positives were not distinguished from the amplification background noise of the negative control (Figure 5B), which introduced false positives into the results. The product of the $E 1$ primers had a weak signal at low-copy number (Figure 5B), introducing false negatives into the results. Furthermore, the RT-PCR method tested was still sensitive to inhibitors present in the environmental samples (data not shown).

Other methods have been developed to detect very small quantities of target sequence. One of these methods is the Rolling Circle Amplification (RCA), whereupon recognition of 
the target sequence, RNA or DNA, by a specific linear probe, a ligase circularizes the template. Using primers designed to hybridize with the probe, a DNA polymerase with stranddisplacement activity amplifies the probe in an isothermal reaction ${ }^{42}$. It is the probe that identified the target, which is amplified, and not the target sequence, which makes this method highly sensitive ${ }^{43}$. Wang et al. ${ }^{44}$ published an RCA protocol for the direct detection of SARS-CoV-1 RNA. The method was modified to use primers specific for SARSCoV-2. Unfortunately, in the non-template control (NTC), the probe circularizes and yields product in the absence of RNA template, even when using a wide variety of ligases, including an SNP-sensitive ligase. In the absence of ligase, the NTC did not show amplification from the linearized probe (Figure 5C).
A

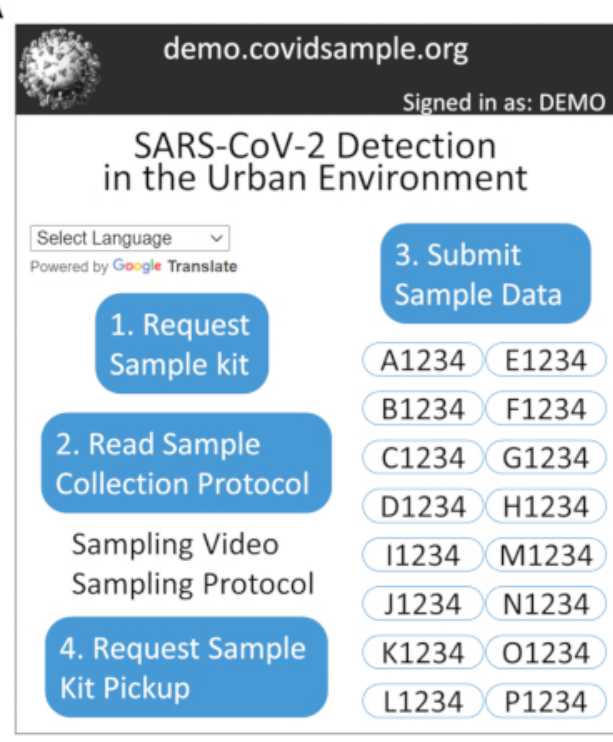

B

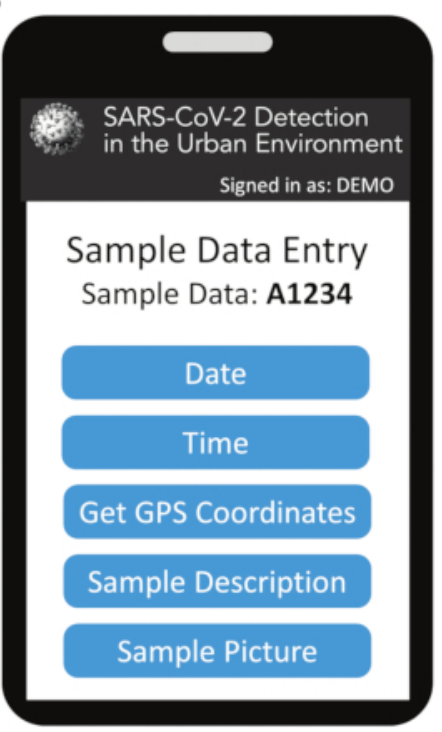

Figure 1: Web-based sampling platform with sample collection data interface for mobile devices. (A) A website, containing a multilingual plugin, was created to mediate the interaction between the laboratory and the citizen scientists. The platform was used for sample kit delivery/pickup request and sample data submission. The platform contained detailed English/Spanish graphic and audiovisual sampling protocols. (B) Mobile device view used to upload sample data: date, time, GPS coordinates, sample site description, and an image of the collection site. Abbreviations: SARS-CoV-2 = severe acute respiratory syndrome coronavirus 2; GPS = Global Positioning System. Please click here to view a larger version of this figure. 


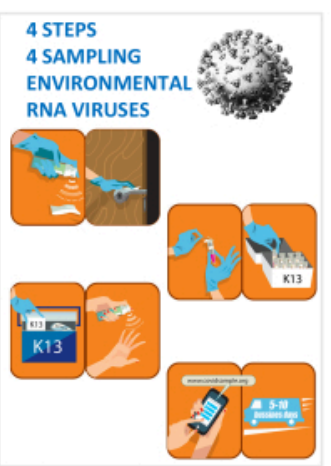

Sampling Protocol

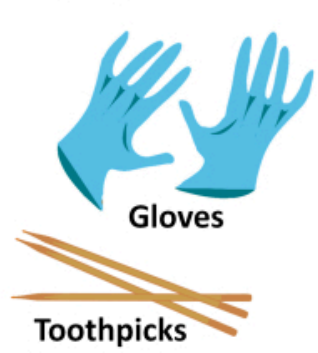

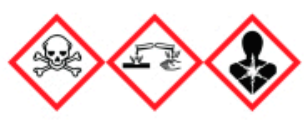

Safety Data Sheet for Trizol

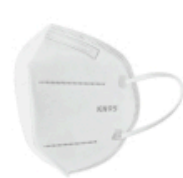

KN95 mask

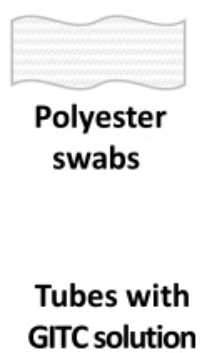

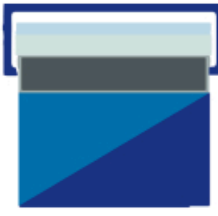

Cooler
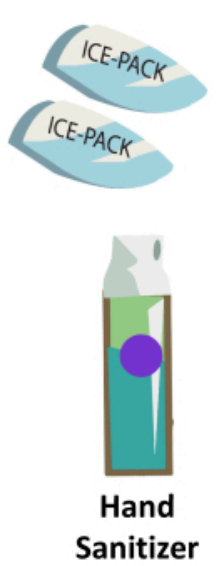

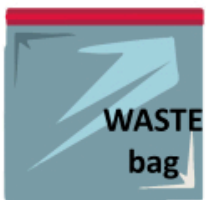

K13

Secondary container

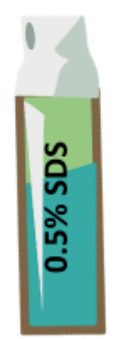

Figure 2: Sample collection kit. Citizen scientists received a cooler containing two ice packs, a safety data sheet to inform volunteers about the hazards of handling the GITC solution, a detailed sampling and mask-wearing protocol, a KN95 mask, a waste bag, a spray bottle with hand sanitizer, a spray bottle with $0.5 \%$ SDS, 16 pairs of gloves, a small bag with 16 toothpicks and 16 polyester swabs, 16 pre-labeled microcentrifuge tubes containing $200 \mu \mathrm{L}$ of GITC solution, a box containing the sampling tubes, and a bag used as the secondary container for the tube box in the event of a spill. Abbreviations: GITC = guanidinium thiocyanate; SDS = sodium dodecyl sulfate. Please click here to view a larger version of this figure. 
A

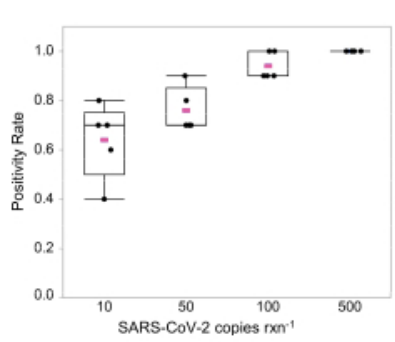

B

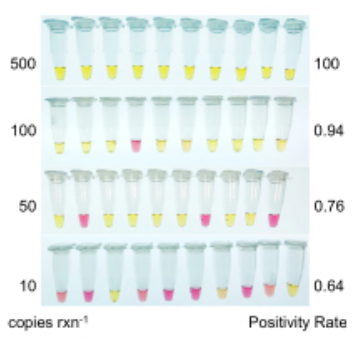

C

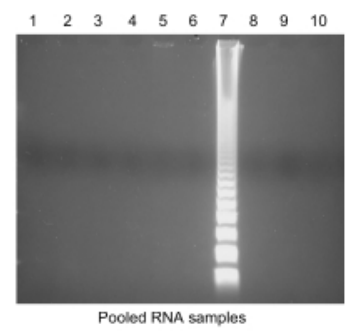

Figure 3: Multiplexed Reverse-Transcription Loop-Mediated Isothermal Amplification (RT-LAMP) assay. Multiplexed reactions using primers for SARS-CoV-2 nucleocapsid (N2) and envelope (E1) genes to detect as few as 10 copies of the virus in the reaction. Synthetic SARS-CoV-2 RNA was used as positive control. (A) Frequency of detection in multiplex colorimetric RT-LAMP of SARS-CoV-2 at different genome copy numbers per reaction. Mean value of five replicates in pink. (B) Limit-of-detection (LOD) of SARS-CoV-2 in multiplex colorimetric RT-LAMP; yellow = positive $(\mathrm{pH} \sim 5)$; pink = negative ( $\mathrm{pH} ~ 8)$. (C) Ladder pattern of positive SARS-CoV-2 RT-LAMP reactions in $1.5 \%$ agarose gel electrophoresis. Abbreviations: SARS-CoV-2 = severe acute respiratory syndrome coronavirus 2; rxn = reaction. Please click here to view a larger version of this figure. 
A

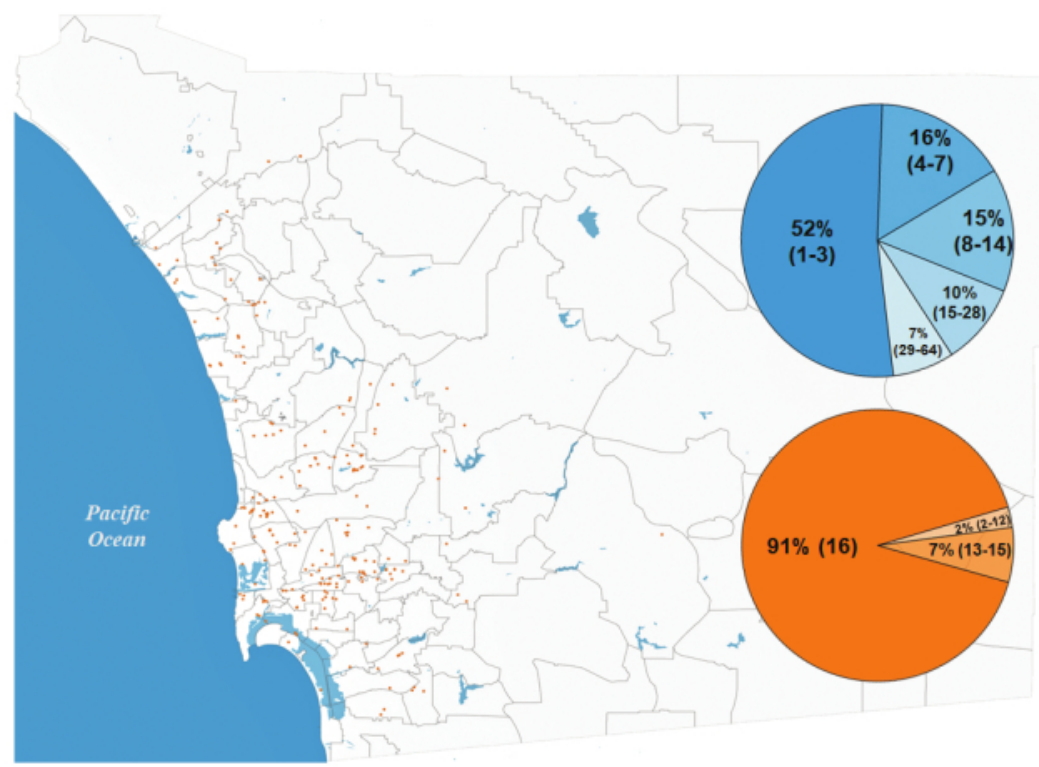

B

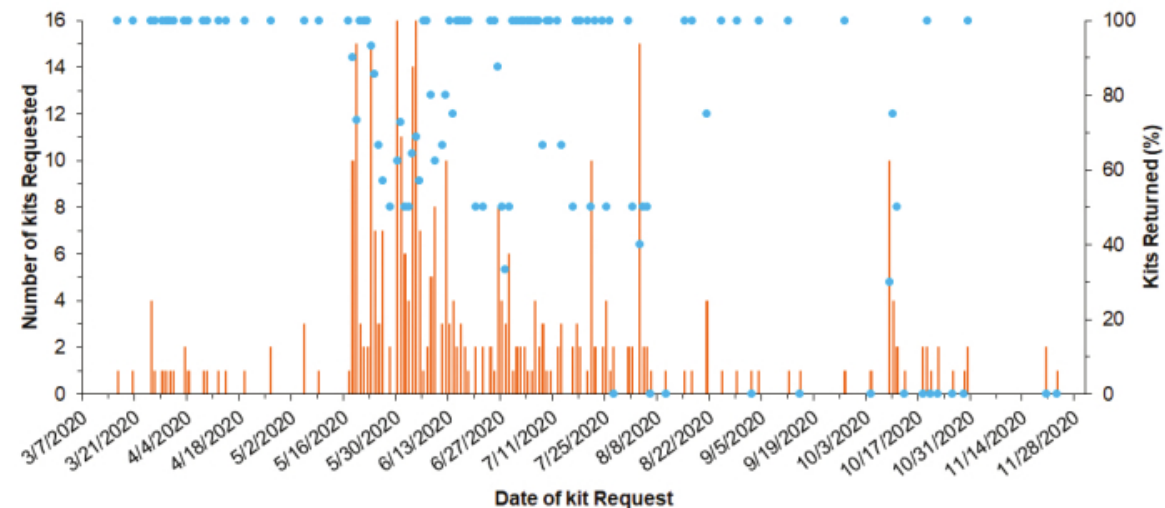

Figure 4: Location of citizen scientist sampling kits in San Diego County and success rate of requested kits.

(A) Orange dots represent the location of 1 sampling kit, which contains 16 samples. The blue pie chart shows the percentage of kits that took various days from when they were delivered to the citizen scientists to when they were returned to the laboratory. Number of days in parentheses. The orange pie chart shows the percentage of kits with different number of completed samples from a total of 16 samples. Number of completed samples containing a swab inside the sample tube and the corresponding sampling data uploaded to the LIMS in parentheses. (B) Percentage of kits that were returned to the laboratory (dots), and total number of requested kits (bars), relative to the date when the kit was requested. Please click here to view a larger version of this figure. 
A

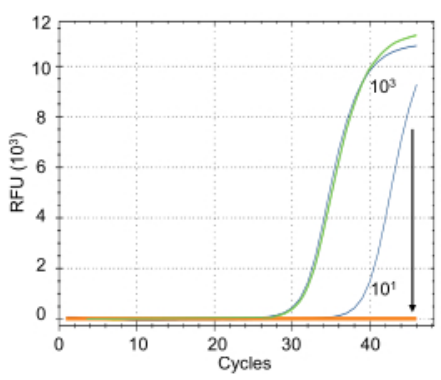

B

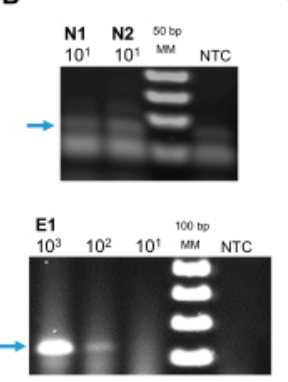

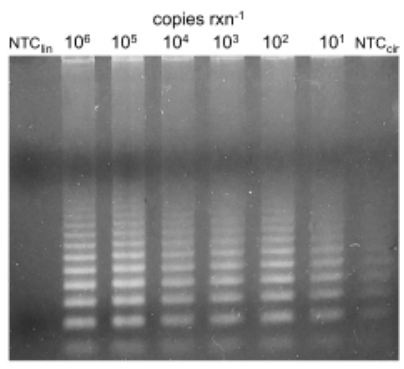

Figure 5: Alternative SARS-CoV-2 RNAdetection methods. (A) RT-qPCR detection of SARS-CoV-2 nucleocapsid $(N)$ gene using primer set N2. Pooled environmental samples spiked with 900 (green), or 9 (orange) copies of SARS-CoV-2. Positive controls of the same copy numbers in blue. Arrow indicates the decrease in fluorescence detection of low-copy number positive control when environmental sample is present. (B) Traditional RT-PCR detection of SARS-CoV-2. Top: RT-PCR products of the nucleocapsid gene using primer sets N1 and N2. A faint background signal is observed in the no-template control. Bottom: RT-PCR products of the envelope gene using primer set E1. Very low signal is observed at the LOD concentration. Blue arrows show expected positive product: (top) 70 bp and (bottom) 113 bp in $2 \%$ agarose gel electrophoresis. (C) RCA of SARS-CoV-2 RNA. Circular probe amplifies in the presence of ligase and absence of RNA template $\left(\mathrm{NTC}_{\mathrm{cir}}\right)$; in the absence of ligase and RNA template linear probe does not amplify (NTClin). Abbreviations: SARSCoV-2 = severe acute respiratory syndrome coronavirus $2 ; \mathrm{RFU}=$ relative fluorescence units; $b p=$ base pairs; rxn = reaction; $\mathrm{MM}$ = molecular marker; RT-PCR = reverse-transcription polymerase chain reaction; RT-qPCR = real-time quantitative RTPCR; RCA = rolling circle amplification; NTC= no-template control; LOD = limit of detection; $r x n=$ reaction. Please click here to view a larger version of this figure. 


\begin{tabular}{|c|c|c|}
\hline Primer & 20X Concentration $(\mu \mathrm{M})$ & 1X Concentration $(\mu \mathrm{M})$ \\
\hline FIP & 32 & 1.6 \\
\hline BIP & 32 & 0.2 \\
\hline F3 & 4 & 0.2 \\
\hline B3 & 4 & 0.4 \\
\hline LoopF & 8 & 0.4 \\
\hline LoopB & 8 & 0.6 \\
\hline
\end{tabular}

Table 1: Formulation for 20X RT-LAMP primer mix. In the RT-LAMP reaction, 6 primers recognize 8 regions of the targeted DNA. Abbreviations: reverse-transcription loop-mediated isothermal amplification; FIP = forward inner primer; BIP = backward inner primer; F3 = forward displacement primer; B3 = backward displacement primer; LoopF = forward loop primer; LoopB = backward loop primer. 


\begin{tabular}{|c|c|c|c|}
\hline Primer & Sequence & Target & Product size \\
\hline \multicolumn{4}{|c|}{ RT-LAMP $9,18,19$} \\
\hline E1-F3 & TGAGTACGAACTTATGTACTCAT & \multirow[t]{6}{*}{$\mathrm{E}$} & \multirow{12}{*}{$\begin{array}{l}\text { ladder- } \\
\text { like pattern }\end{array}$} \\
\hline E1-B3 & TTCAGATTTTTAACACGAGAGT & & \\
\hline E1-FIP & ACCACGAAAGCAAGAAAAAGAAGTTCGTTTCGGAAGAGACAG & & \\
\hline E1-BIP & TTGCTAGTTACACTAGCCATCCTTAGGTTTTACAAGACTCACGT & & \\
\hline E1-LoopB & GCGCTTCGATTGTGTGCGT & & \\
\hline E1-LoopF & CGCTATTAACTATTAACG & & \\
\hline N2-F3 & ACCAGGAACTAATCAGACAAG & \multirow[t]{6}{*}{$\mathrm{N}$} & \\
\hline N2-B3 & GACTTGATCTTTGAAATTTGGATCT & & \\
\hline N2-FIP & TTCCGAAGAACGCTGAAGCGGAACTGATTACAAACATTGGCC & & \\
\hline N2-BIP & CGCATTGGCATGGAAGTCACAATTTGATGGCACCTGTGTA & & \\
\hline N2-LoopF & GGGGGCAAATTGTGCAATTTG & & \\
\hline N2-LoopB & CTTCGGGAACGTGGTTGACC & & \\
\hline \multicolumn{4}{|c|}{ RT-qPCR 38} \\
\hline $\begin{array}{c}\text { 2019- } \\
\text { nCoV_N1-F }\end{array}$ & GACCCCAAAATCAGCGAAAT & \multirow[t]{3}{*}{$\mathrm{N}$} & \multirow[t]{3}{*}{$72 \mathrm{bp}$} \\
\hline $\begin{array}{c}\text { 2019- } \\
\text { nCoV_N1-R }\end{array}$ & TCTGGTTACTGCCAGTTGAATCTG & & \\
\hline $\begin{array}{c}\text { 2019- } \\
\text { nCoV_N1-P }\end{array}$ & 5'-FAM-ACC CCG CAT TAC GTT TGG TGG ACC-BHQ1-3' & & \\
\hline $\begin{array}{c}\text { 2019- } \\
\text { nCoV_N2-F }\end{array}$ & TTACAAACATTGGCCGCAAA & \multirow[t]{3}{*}{$\mathrm{N}$} & \multirow[t]{3}{*}{$67 \mathrm{bp}$} \\
\hline $\begin{array}{c}\text { 2019- } \\
\text { nCoV_N2-R }\end{array}$ & GCGCGACATTCCGAAGAA & & \\
\hline $\begin{array}{c}\text { 2019- } \\
\text { nCoV_N2-P }\end{array}$ & 5'-FAM-ACA ATT TGC CCC CAG CGC TTC AG-BHQ1-3' & & \\
\hline \multicolumn{4}{|c|}{ RT-PCR 39} \\
\hline
\end{tabular}




\begin{tabular}{|c|c|c|c|}
\hline E1_Sarbeco_F & ACAGGTACGTTAATAGTTAATAGCGT & E & $113 \mathrm{bp}$ \\
\cline { 1 - 2 } E1_Sarbeco_R & ATATTGCAGCAGTACGCACACA & & \\
\hline
\end{tabular}

Table 2: Primers used for RT-LAMP, RT-qPCR, and RT-PCR. Primer sequences, target gene, expected product size, and corresponding reference are listed. Abbreviations: RT-LAMP = reverse-transcription loop-mediated isothermal amplification; $\mathrm{RT}-\mathrm{PCR}$ = reverse-transcription polymerase chain reaction; $\mathrm{bp}=$ base pairs; RT-qPCR = real-time quantitative RT-PCR; E1 = envelope gene; $N 2$ = nucleocapsid gene; $F=$ forward primer; $R=$ reverse primer; $P=$ Probe $; F I P=$ forward inner primer; $\mathrm{BIP}=$ backward inner primer; F3 = forward displacement primer; B3 = backward displacement primer; LoopF = forward loop primer; LoopB = backward loop primer.

\begin{tabular}{|c|c|}
\hline Reagent & Volume $(\mu \mathrm{L})$ \\
\hline WarmStart Colorimetric LAMP 2X Master Mix with UDG & 12.5 \\
\hline N2 Primer Mix (20x) & 1.25 \\
\hline E1 Primer Mix (20x) & 1.25 \\
\hline Guanidine Hydrochloride $(600 \mathrm{mM})^{*}$ & 2.5 \\
\hline Target RNA & 5 \\
\hline Nuclease-free $\mathrm{H}_{2} \mathrm{O}$ & 2.5 \\
\hline Total Volume & 25 \\
\hline
\end{tabular}

Table 3: Reaction master mix for multiplex colorimetric RT-LAMP. ( $\left.{ }^{*}\right)$ Guanidine Hydrochloride has been shown to increase the sensitivity and speed of the reaction by an uncharacterized mechanism ${ }^{28}$. Abbreviations: LAMP $=$ loopmediated isothermal amplification; UDG = uracil-DNA glycosylase; $N 2$ = nucleocapsid gene; $E 1$ = envelope gene; DEPC = diethylpyrocarbonate. 


\begin{tabular}{|c|c|c|c|c|c|c|c|c|}
\hline $\begin{array}{c}\text { Approved } \\
\text { citizens }\end{array}$ & $\begin{array}{c}\text { Citizens } \\
\text { who }\end{array}$ & $\begin{array}{c}\text { Delivered } \\
\text { kits }\end{array}$ & $\begin{array}{c}\text { Returned } \\
\text { sampled kits }\end{array}$ & \multicolumn{2}{|c|}{$\begin{array}{c}\text { Days dedicated } \\
\text { to sampling }\end{array}$} & $\begin{array}{c}\% \text { Complete } \\
\text { kits }\end{array}$ & $\begin{array}{c}\% \\
\text { Incomplete }\end{array}$ & $\begin{array}{c}\text { Processed } \\
\text { samples }\end{array}$ \\
\hline \multirow[t]{2}{*}{482} & \multirow{2}{*}{$\begin{array}{c}72.6 \% \\
(350 / 482)\end{array}$} & \multirow[t]{2}{*}{362} & \multirow{2}{*}{$\begin{array}{c}70.4 \% \\
(255 / 362)\end{array}$} & Mean & 8 & \multirow{2}{*}{$\begin{array}{c}91.1 \\
(224 / 246)\end{array}$} & \multirow[t]{2}{*}{$8.9(22 / 246)$} & \multirow[t]{2}{*}{4,080} \\
\hline & & & & Median & 3 & & & \\
\hline
\end{tabular}

Table 4:Swabbing for SARS-CoV-2 by the numbers. Outreach and sampling success rates. Abbreviation: SARS-CoV-2 = severe acute respiratory syndrome coronavirus 2 .

\section{Discussion}

Citizen scientist engagement. Citizen scientists were recruited to swab surfaces throughout San Diego County to sample and detect the presence of SARS-CoV-2 in the urban environment. The majority of delivered sampling kits $(70 \%)$ were returned to the laboratory, and of those, almost all samples were complete (91\%) (Figure 3A,B and Table 4). Volunteers could easily request kit delivery/pickup through the web-based platform, and the delivery route-planning software notified citizen scientists of estimated times of arrival, both likely significant factors for the observed success. The average time from when the kit was delivered to the citizen scientist to when it was returned to the laboratory was 8 days, with a median of 3 days and a range of 1-64 days (Figure 3A and Table 4). More frequent reminders to volunteers would likely reduce this lag time.

The data collection platform was successfully used by a great majority of users $(73 \%)$ (Table 4$)$. While the efforts of the citizen scientists were not measured, field tests showed that the data collection platform significantly reduced the effort and time required to properly complete sample collection. Thus, reducing the amount of bookkeeping encouraged citizen scientist engagement. The web-based platform intended to overcome demographic limitations by providing a multilingual neural machine translation service and by providing graphic and audiovisual protocols in English and Spanish. This was only partially successful as fewer samples were collected from both the South Bay and North County where most of the county's Hispanic/Latino population reside ${ }^{45}$. These areas also harbored $63 \%(1,700$ cases per 100,000$)$ of the total COVID-19 cases in San Diego County with the highest prevalence of the disease ${ }^{46}$ and rate of hospitalizations $(62 \%)^{47,48}$. Although most of the samples came from Central County, a representative number was collected from the most COVID-19-impacted districts and only a small fraction of the samples was positive, which suggests that surface reservoirs of SARS-CoV-2 in the urban environment are relatively rare.

Sample processing. Sampling swabs were wetted with SDS, which inactivated the virus by disrupting its envelope and stabilized the naked RNA by unfolding RNases ${ }^{32}$. Conveniently during collection, the detergent in the swab cleaned the sampled surface. Environmental samples often contain very small quantities of RNA. To maximize recovery, RNA isolation was performed using a GITC-based, columnfree, crude extraction method. GITC, a strong chaotropic agent, disrupts the hydrogen bonds that maintain protein folding (i.e., hydrophobic effect). This action results in the 
inactivation of viral particles, and the RNA remains stable due to the inhibition of RNAses ${ }^{34,35,36}$. The GITC solution maintained the stability of the RNA samples without strict cold-chain considerations, which allowed the citizens to maintain the samples at room temperature if a freezer for the provided ice packs was not available. To reduce the potential hazard this reagent poses when direct skin or mucosal contact occurs, citizens were made aware of these risks by the inclusion of a material safety data sheet provided in the kit and a warning seal was placed in the box containing the tubes.

The crude GITC-chloroform extraction method aided the recovery of traces of RNA from the swabs, and as shown by the amplification of spiked samples, inhibitors rarely persisted in the samples after extraction. Samples, which were negative for SARS-CoV-2 and showed no RT-LAMP inhibition, represented true negatives or had a lower copy number than the LOD at $100 \%$ frequency. Conversely, detection of viral RNA on a surface does not directly imply risk of transmission through contact as the infectivity of the virus from positive samples needs to be tested. Prompt screening of the environment, not limited by the availability of sophisticated supplies or highly qualified personnel, is crucial to assess whether surfaces constitute a viral reservoir and to better direct prevention and containment efforts.

RT-LAMP was selected to be the best method suitable for the proposed detection pipeline. It proved to be a rapid and inexpensive method that was highly resistant to most of the remaining inhibitors and as sensitive and specific as other RTqPCR methods. Because of their use in clinical settings during the SARS-CoV-2 pandemic, the availability of RT-qPCR kits was impacted by global demand. Moreover, RT-qPCR techniques-even those formulated to resist inhibitors- were sensitive to substances contained in the environmental samples collected by a pilot cohort of citizen scientists, even after the use of other common strategies to reduce inhibitor competition for enzyme binding ${ }^{49}$. These findings are corroborated by a recent study that compared both methods to detect SARS-CoV-2 on swab samples from candy handled by COVID-19 patients and found over $83 \%$ result concordance, with $25 \%$ lower inhibition in samples analyzed by RT-LAMP ${ }^{15}$. Furthermore, the GITC-chloroform crude extraction, coupled with RT-LAMP, reduced the cost of reagents and supplies by $42 \%$ compared to RNA kit extraction and RT-qPCR (Table of Materials).

This method allowed for high throughput analysis of thousands of surface swab samples. Up to 80 pools, representing 640 samples, were processed in 2 days from RNA extraction to SARS-CoV-2 detection by RT-LAMP. The proposed protocol is semiquantitative, limited to the detection of viral RNA, and does not indicate the presence of infective viral particles. Further analysis is required to assess the risk of transmission of SARS-CoV-2 from infected fomites present at the swabbed surfaces.

This study presents a protocol to quickly set up a testing strategy that includes an effective workflow when facing a health emergency with a communicable disease. The proposed sampling protocol is simple and uses supplies commonly found in households, and the viral detection method is carried out on equipment available in basic laboratory settings such as a water bath in lieu of a thermocycler. The costs of RT-LAMP reagents are significantly lower than those needed for RT-qPCR and less susceptible to high global demand scenarios. This study serves as a framework for the assessment of environmental 
viral reservoirs in future epidemic outbreaks and global pandemics.

\section{Disclosures}

All authors declare that no competing interests exist.

\section{Acknowledgments}

We thank the Viral Information Institute (VII) investigators, Dr. Anca M. Segall, Willow Segall, Patricia L. Rohwer, Gary Rohwer, Cary L. Rohwer, Magda Silvia Pinetta, Elizabeth Cruz Cano, Dr. Gregory Peters, Dr. Stuart A. Sandin, and Dr. Jennifer Smith for taking the time to collect numerous samples. We also thank Dr. Rob Knight, Dr. Jack Gilbert, Dr. Pedro Balda-Ferre, and Dr. Sarah Allard from the department of Pediatrics at the School of Medicine University of San Diego California (UCSD) for facilitating positive controls and useful feedback. We thank Stacey Carota (SDSU College of Sciences) and Gina Spidel (SDSU) for logistical support and Juan Rodríguez for the art and graphic design of the sampling protocol. We thank all participants for their commitment and dedication to this project during very difficult times. This work was supported by a generous donation from Dr. Jo Ann Lane (SDSU College of Sciences) and the National Science Foundation RAPID: Environmental Reservoirs of SARS-CoV-2 grant (Award Number: 2030479).

\section{References}

1. Alsved, M. et al. Exhaled respiratory particles during singing and talking. Aerosol Science and Technology. 54 (11), 1245-1248 (2020).

2. Morawska, L., Cao, J. Airborne transmission of SARSCoV-2: The world should face the reality. Environment International. 139, 105730 (2020).
3. Stadnytskyi, V., Bax, C. E., Bax, A., Anfinrud, P. The airborne lifetime of small speech droplets and their potential importance in SARS-CoV-2 transmission. Proceedings of the National Academy of Sciences of the United States of America. 117 (22), 11875-11877 (2020).

4. Yu, I. T. S. et al. Evidence of Airborne Transmission of the Severe Acute Respiratory Syndrome Virus. New England Journal of Medicine. 350 (17), 1731-1739 (2004).

5. World Health Organization. Coronavirus disease (COVID-19): How is it transmitted? https://www.who.int/ emergencies/diseases/novel-coronavirus-2019/ question-and-answers-hub/q-a-detail/coronavirusdisease-covid-19-how-is-it-transmitted (2020).

6. Centers for Disease Control and Prevention. How COVID-19 Spreads. https://www.cdc.gov/ coronavirus/2019-ncov/prevent-getting-sick/how-covidspreads.html (2020).

7. Moriarty, L. F. et al. Public Health Responses to COVID-19 Outbreaks on Cruise Ships - Worldwide, February-March 2020. MMWR. Morbidity and Mortality Weekly Report. 69 (12), 347-352 (2020).

8. Cheng, V. C.-C. et al. Air and environmental sampling for SARS-CoV-2 around hospitalized patients with coronavirus disease 2019 (COVID-19). Infection Control and Hospital Epidemiology. 1-8 (2020).

9. Van Doremalen, N. et al. Aerosol and surface stability of SARS-CoV-2 as compared with SARS-CoV-1. New England Journal of Medicine. 382 (16), 1564-1567 (2020).

10. Liu, Y. et al. Aerodynamic analysis of SARS-CoV-2 in two Wuhan hospitals. Nature. 582, 557-560 (2020). 
11. Butler, D. J. et al. Shotgun transcriptome and isothermal profiling of SARS-CoV-2 infection reveals unique host responses, viral diversification, and drug interactions. bioRxiv. (2020).

12. Döhla, M. et al. SARS-CoV-2 in environmental samples of quarantined households. medRxiv. (2020).

13. Ikonen, N. et al. Deposition of respiratory virus pathogens on frequently touched surfaces at airports. BMC Infectious Diseases. 18, 437 (2018).

14. Chia, P. Y. et al. Detection of air and surface contamination by SARS-CoV-2 in hospital rooms of infected patients. Nature Communications. 11 (1), 2800 (2020).

15. Salido, R. A. et al. Handwashing and detergent treatment greatly reduce SARS-CoV-2 viral load on Halloween candy handled by COVID-19 patients. mSystems. 5, e01074-20 (2020).

16. Chan, J. F. W. et al. Improved molecular diagnosis of COVID-19 by the novel, highly sensitive and specific COVID-19-RdRp/Hel real-time reverse transcriptionPCR assay validated in vitro and with clinical specimens. Journal of Clinical Microbiology. 58 (5), e00310-20 (2020).

17. Dao Thi, V. L. et al. A colorimetric RT-LAMP assay and LAMP-sequencing for detecting SARS-CoV-2 RNA in clinical samples. Science Translational Medicine. 12 (556), eabc7075 (2020).

18. Rauch, J. et al. A scalable, easy-to-deploy, protocol for Cas13-based detection of SARS-CoV-2 genetic material. bioRxiv. (2020).

19. Zhang, F., Abudayyeh, O. O., Gootenberg, J. S. A protocol for detection of COVID-19 using
CRISPR diagnostics. https://www.broadinstitute.org/ files/publications/special/COVID-19\%20detection \%20(updated).pdf. (2020).

20. Zhang, Y. et al. Rapid molecular detection of SARSCoV-2 (COVID-19) virus RNA using colorimetric LAMP. medRxiv. (2020).

21. Zhang, Y. et al. Enhancing colorimetric loop-mediated isothermal amplification speed and sensitivity with guanidine chloride. BioTechniques. 69 (3), 179-185 (2020).

22. Broughton, J. P. et al. CRISPR-Cas12-based detection of SARS-CoV-2. Nature Biotechnology. 38, 870-874 (2020).

23. Lucia, C., Federico, P.-B., Alejandra, G. C. An ultrasensitive, rapid, and portable coronavirus SARSCoV-2 sequence detection method based on CRISPRCas12. bioRxiv. (2020).

24. Danko, D. et al. Global genetic cartography of urban metagenomes and anti-microbial resistance. bioRxiv. (2020).

25. Parida, M. et al. Rapid detection and differentiation of dengue virus serotypes by a real-time reverse transcription-loop-mediated isothermal amplification assay. Journal of Clinical Microbiology. 43 (6), 2895-2903 (2005).

26. Notomi, T. et al. Loop-mediated isothermal amplification of DNA. Nucleic acids research. 28 (12), e63 (2000).

27. Nagamine, K., Hase, T., Notomi, T. Accelerated reaction by loop-mediated isothermal amplification using loop primers. Molecular and Cellular Probes. 16 (3), 223-229 (2002). 
28. Zhang, Y. et al. Enhancing colorimetric loop-mediated isothermal amplification speed and sensitivity with guanidine chloride. BioTechniques. 69 (3), 179-186 (2020).

29. Tanner, N. A., Zhang, Y., Evans, T. C. Visual detection of isothermal nucleic acid amplification using $\mathrm{pH}$-sensitive dyes. BioTechniques. 58 (2), 59-68 (2015).

30. Curtis, K. A., Rudolph, D. L., Owen, S. M. Rapid detection of HIV-1 by reverse-transcription, loopmediated isothermal amplification (RT-LAMP). Journal of Virological Methods. 151 (2), 264-270 (2008).

31. Internet Engineering Task Force (IETF). OAuth 2.0. https://oauth.net/2/ (2012).

32. Naidu, K. T., Prabhu, N. P. Protein-surfactant interaction: Sodium dodecyl sulfate-induced unfolding of ribonuclease A. Journal of Physical Chemistry B. 115 (49), 14760-14767 (2011).

33. Chomczynski, P., Sacchi, N. Single-step method of RNA isolation by acid guanidinium thiocyanate-phenolchloroform extraction. Analytical Biochemistry. 162 (1), 156-159 (1987).

34. Chomczynski, P., Sacchi, N. The single-step method of RNA isolation by acid guanidinium thiocyanatephenol-chloroform extraction: Twenty-something years on. Nature Protocols. 1 (2), 581-585 (2006).

35. Hummon, A. B., Lim, S. R., Difilippantonio, M. J., Ried, T. Isolation and solubilization of proteins after TRIzol ${ }^{\circ}$ extraction of RNA and DNA from patient material following prolonged storage. Biotechniques. 42 (4), 467-472 (2007).
36. Rio, D. C., Ares, M., Hannon, G. J., Nilsen, T. W. Purification of RNA using TRIzol (TRI Reagent). Cold Spring Harbor Protocols. (2010).

37. Rio, D. C., Ares, M., Hannon, G. J., Nilsen, T. W. Ethanol precipitation of RNA and the use of carriers. Cold Spring Harbor Protocols. (2010).

38. Wallace, D. M. Precipitation of nucleic acids. Methods in Enzymology. 152, 41-48 (1987).

39. County of San Diego Health and Human Services Agency. COVID-19 Dashboard. https://www.arcgis.com/ apps/opsdashboard/ index.html\#/96feda77f12f46638b984fcb1d17bd24 (2020).

40. Centers for Disease Control and Prevention. CDC 2019-novel Coronavirus (2019-nCoV) real-time RT-PCR diagnostic panel. https://www.fda.gov/media/134922/ download (2020).

41. Corman, V. M. et al. Detection of 2019 novel coronavirus (2019-nCoV) by real-time RT-PCR. Eurosurveillance. 25 (3), 2000045 (2020).

42. Lizardi, P. M. et al. Mutation detection and single-molecule counting using isothermal rolling-circle amplification. Nature Genetics. 19, 225-232 (1998).

43. Johne, R., Müller, H., Rector, A., van Ranst, M., Stevens, H. Rolling-circle amplification of viral DNA genomes using phi29 polymerase. Trends in Microbiology. 17 (5), 205-211 (2009).

44. Wang, B. et al. Rapid and sensitive detection of severe acute respiratory syndrome coronavirus by rolling circle amplification. Journal of Clinical Microbiology. 43 (5), 2339-2344 (2005). 
45. County of San Diego Health and Human Services Agency. Population of Mexican origin in San Diego County. https://www.sandiegocounty.gov/content/dam/ sdc/hhsa/programs/phs/CHS/ENGLISH

VERSION_Mexican Origin.pdf (2020).

46. County of San Diego Health and Human Services Agency. COVID-19 city of residence MAP. https://www.sandiegocounty.gov/content/dam/ sdc/hhsa/programs/phs/Epidemiology/COVID-19 City of Residence_MAP.pdf (2020).

47. County of San Diego Health and Human Services Agency. COVID-19 hospitalizations summary. https://www.sandiegocounty.gov/content/ dam/sdc/hhsa/programs/phs/ Epidemiology/COVID-19 Hospitalizations Summary_ALL.pdf (2020).

48. County of San Diego Health and Human Services Agency. COVID-19 race and ethnicity Summary. https://www.sandiegocounty.gov/content/ dam/sdc/hhsa/programs/ phs/Epidemiology/COVID-19 Race and Ethnicity Summary.pdf (2020).

49. Schrader, C., Schielke, A., Ellerbroek, L., Johne, R. PCR inhibitors - occurrence, properties and removal. Journal of Applied Microbiology. 113 (5), 1014-1026 (2012). 